\title{
Nocturnal Changes in Knee Cartilage Thickness in Young Healthy Adults
}

\author{
K.H. Sitoci ${ }^{a} \quad$ M. Hudelmaier ${ }^{b} \quad$ F. Eckstein ${ }^{b}$ \\ ${ }^{a}$ Musculoskeletal Research Group, Institute of Anatomy, Ludwig-Maximilians-Universität München, Munich,

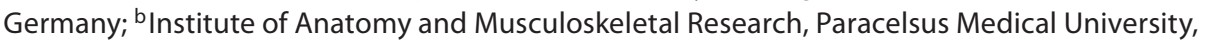 \\ Salzburg, Austria
}

\section{Key Words}

Cartilage $\cdot$ Knee joint $\cdot$ Deformation $\cdot$ Magnetic resonance imaging

\begin{abstract}
Magnetic resonance imaging (MRI) allows one to analyze cartilage physiology in vivo. Cartilage deforms during loading, but little is known about its recovery after deformation. Here we study 'nocturnal' changes in knee cartilage thickness and whether postexercise deformation differs between morning and evening. Axial magnetic resonance (MR) images were acquired in the right knees of 17 healthy volunteers (age $23.5 \pm 3.0$ years) after a normal day, and then after 30 deep knee bends. Coronal images were additionally acquired in 8 of these volunteers after a normal day and then after 2 min of static loading of the leg with 150\% body weight. The volunteers then remained unloaded overnight and the same protocol was repeated in the morning. A significant increase $(p<0.01)$ in cartilage thickness was observed between evening (preexercise) and morning (preexercise): $+2.4 \%$ in the patella, $+8.4 \%$ in the medial tibia and $+6.2 \%$ in the lateral tibia. Deformation in the morning (-6.8/ $-4.6 /-5.1 \%)$ was generally greater than that in the evening $(-5.4 /-3.2 /-3.7 \%)$, but this difference did not reach statistical significance. No significant difference in the nocturnal thickness increase (or postexercise deformation) was observed between men and women. We conclude that knee cartilage
\end{abstract}

(thickness) recovers overnight by approximately $2-8 \%$, independent of sex. Given the lack of 'predeformation' after nocturnal periods of unloading, morning postexercise deformation of the cartilage may have a greater magnitude than evening postexercise deformation.

Copyright $\odot 2012$ S. Karger AG, Basel

\section{Introduction}

The metabolism of chondrocytes is known to react to changes in the biomechanical environment [Sah et al., 1989; Urban, 1994; Kim et al., 1995; Wong et al., 1997; Quinn et al., 1998; Buschmann et al., 1999; Ofek et al., 2010] and knowledge on mechanical tissue 'strains' occurring 'in vivo' can thus enhance the understanding of mechanobiological interactions. Such knowledge can also guide mechanical cell stimulation of tissue culture (i.e. bioreactor). In vitro/ex vivo studies only provide an

\begin{tabular}{ll} 
Abbreviations used in this paper \\
\hline BMI & body mass index \\
MR & magnetic resonance \\
MRI & magnetic resonance imaging
\end{tabular}


approximation of the in vivo deformation of cartilage since the joint loads occurring in vivo are largely unknown and because joint pressure is unevenly distributed throughout cartilage surfaces.

Magnetic resonance imaging (MRI) permits the noninvasive measurement of deformational behavior in situ and in vivo [Eckstein et al., 2001, 2006b]. Under in situ (ex vivo) conditions, patellar cartilage deformed little during the first minutes of static loading [Herberhold et al., 1999] due to hydrostatic pressurization of the tissue [Ateshian et al., 1994]. Even after several hours of loading, the cartilage continued to deform without reaching equilibrium and recovery from static loading required several hours [Herberhold et al., 1999]. In vivo deformation of the patellar cartilage amounted to 5-6\% after deep knee bends [Eckstein et al., 1998], with a period of $90 \mathrm{~min}$ being required to recover from deformation [Eckstein et al., 1999]. A 'dose-dependent' magnitude of patellar cartilage deformation was observed after various physiological activities that scaled with assumed load severity [Eckstein et al., 2005]. In vivo deformation of femorotibial cartilage, however, has been studied to a lesser extent and the magnitude of deformation observed was smaller than that for patellar cartilage under similar loading, i.e. knee bends, and a somewhat more homogeneous response (across participants) was seen under static loading [Eckstein et al., 2005].

Waterton et al. [2000] showed that no significant diurnal changes occur in healthy femoral cartilage volume, but subtle differences in the regional distribution of femoral cartilage thickness were described between the morning and evening. However, because the participants of the above study walked to the MRI unit in the morning and deformation may have occurred between getting up and the MR image acquisition, this observation does not preclude that recovery (thickness increase) of knee cartilage may occur overnight from mechanical loading during the day. MRI has previously been used to study nocturnal vertebral volume (and fluid flow) in humans [Paajanen et al., 1994; Roberts et al., 1998; Malko et al., 2002; Hutton et al., 2003], but, to our knowledge, the nocturnal changes of healthy cartilage have not yet been studied. The purpose of this study, therefore, was to employ MRI in vivo in order to explore:

- The magnitude of 'nocturnal' changes in knee (patellar and tibial) cartilage thickness, i.e. differences in cartilage thickness after a normal day and physiological unloading overnight

- Whether postexercise deformation of the cartilage is greater in the morning (after a long period of unloading) than in the evening (after a normal day)
- Whether there are sex differences in the nocturnal cartilage thickness changes, or in postexercise deformation between men and women

\section{Methods}

The right knees of 17 healthy young subjects were examined. Eight volunteers were female (age [mean \pm SD] $22 \pm 2.7$ years, body mass index (BMI) $22.23 \pm 2.20$ ) and nine male (age [mean $\pm \mathrm{SD}] 24.3 \pm 3.2$ years, BMI $21.5 \pm 2.1$ ). The volunteers had no history of symptoms, signs, trauma or surgery at the knee joint, had a normal BMI $(<25)$ and were not professional or amateur athletes. Leg dominance and knee alignment were not determined. The study was ratified by the local ethics committee (University of Munich). Written informed consent was obtained from all volunteers prior to the study.

Axial MR images of all participants were obtained at $1.5 \mathrm{~T}$ (Magnetom Vision; Siemens, Erlangen, Germany), using a circumferentially polarized knee coil. The idea of also studying the femorotibial joint emerged throughout the study and coronal MR images were additionally obtained in the last 8 volunteers recruited. Of these, 4 were women (age $23.3 \pm 1.3$ years, BMI $23.8 \pm$ 0.90 ) and 4 men (age $25.0 \pm 3.0$ years, BMI $22.5 \pm 2.6$ ). Image acquisition was performed using a $3 \mathrm{D}_{1}$-weighted $3 \mathrm{D}$-FLASH sequence with water excitation (repetition time $=17.2 \mathrm{~ms}$, echo time $=6.6 \mathrm{~ms}$, flip angle $=20^{\circ}$, slice thickness $=1.5 \mathrm{~mm}$, in-plane resolution $=0.31 \times 0.31 \mathrm{~mm}$, axial acquisition time $=3 \mathrm{~h} 47 \mathrm{~min}$, coronal acquisition time $=6 \mathrm{~h} 19 \mathrm{~min}$ [Eckstein et al., 2005]) that has been previously validated for the purpose of cartilage thickness measurement [Graichen et al., 2004]. No fluid-sensitive sequences were acquired to exclude potential bone marrow, meniscus or early cartilage lesions.

The participants were invited to the MRI unit at 8 p.m. after a normal weekday. They had been instructed not to perform strenuous physical activity or sports they were unfamiliar with in the $48 \mathrm{~h}$ preceding data acquisition. After arrival, axial (and coronal) baseline data sets of the right knee were obtained. The knee was then repositioned in the scanner and a second axial (and coronal) acquisition was performed. The participants were then asked to perform 30 deep knee bends (up to $120^{\circ}$ knee angle) within $90 \mathrm{~s}$ outside the MRI scanner; they were then immediately repositioned in the magnet and another axial acquisition was obtained at $90 \mathrm{~s}$ after finishing the knee bends. Eight volunteers were additionally asked to then stand on their right leg on a commercial bathroom scale for $120 \mathrm{~s}$, with the knee flexed to $15^{\circ}$ [Eckstein et al., 2005]. Weights equivalent to $50 \%$ of their body weight were added to their back (i.e. one-legged stance with $150 \%$ body weight). The knee was then again positioned in the magnet and the postloading coronal data acquisition was started $90 \mathrm{~s}$ after the static loading exercise. All volunteers then spent the night at the MRI unit and were not allowed to stand up or walk. When the volunteers had to use the toilet, they were taken by wheelchair and were helped not to step on the right leg. At approximately 5 a.m., after a minimum of an 8-hour nocturnal pause, the same protocol was applied again, starting with two baseline scans (with repositioning in between) and one axial (and coronal) postexercise scan.

Segmentation of the patellar (axial images) and medial and lateral tibial cartilages (coronal images) was performed by one 
Table 1. Nocturnal changes in knee cartilage thickness (pre- and postexercise)

\begin{tabular}{llll}
\hline & $\begin{array}{l}\text { Pre-ex. evening vs. } \\
\text { pre-ex. morning }\end{array}$ & $\begin{array}{l}\text { Post-ex. evening vs. } \\
\text { pre-ex. evening }\end{array}$ & $\begin{array}{l}\text { Post-ex. morning vs. } \\
\text { pre-ex. morning }\end{array}$ \\
\hline P & $+2.4 \pm 2.2 \%^{* * *}$ & $-5.4 \pm 2.2 \%^{* * *}$ & $-6.8 \pm 2.7 \%^{* * *}$ \\
MT & $+8.4 \pm 2.6 \%^{* * *}$ & $-3.2 \pm 3.4 \%^{*}$ & $-4.6 \pm 2.6 \%^{* *}$ \\
LT & $+6.2 \pm 3.2 \%^{* *}$ & $-3.7 \pm 1.6 \%^{* * *}$ & $-5.1 \pm 3.5 \%^{* *}$ \\
\hline
\end{tabular}

$\mathrm{P}=$ Patella; $\mathrm{MT}=$ medial tibia $; \mathrm{LT}=$ lateral tibia ex. = exercise (30 deep knee bends in case of the patella, and stance on one leg for 2 min with $150 \%$ body weight in case of the tibia). ${ }^{*} \mathrm{p}<0.05$; ${ }^{* *} \mathrm{p}<0.01 ;{ }^{* *} \mathrm{p}<0.001$.

user (K.H.S.), who had received formal training in cartilage segmentation before starting the study. Image analysis was performed using an Octane Duo workstation (Silicon Graphics, Mountain View, Calif., USA), using a semiautomated segmentation algorithm [Stammberger et al., 1999b]. The readings were done independently for each image, i.e. without uploading other images as a reference. The mean cartilage thickness was determined for each cartilage plate using triangulation and Euclidean distance transformation [Stammberger et al., 1999a].

Test-retest precision for independent analysis or repositioned image acquisitions was determined from the preexercise rest-retest scans (in the evening and morning, respectively), by calculating the root mean square coefficient of variation [Glüer et al., 1995]. This was $1.5 / 1.8 \%$ (evening/morning) in the patella, $2.3 / 1.9 \%$ in the medial tibia and $2.3 / 2.4 \%$ in the lateral tibia.

The magnitude of cartilage deformation after exercise was determined as:

$$
\begin{aligned}
& {\left[\left(\text { Th. }_{1 \text { preexercise }}+\text { Th. } C_{2 \text { preexercise }}\right) / 2\right]-\text { Th. } C_{\text {postexercise }} /} \\
& {\left[\left(\text { Th. } C_{1 \text { preexercise }}+\text { Th. } C_{2 \text { preexercise }}\right) / 2\right] \cdot 100(\%) .}
\end{aligned}
$$

The statistical significance of differences in cartilage thickness was assessed using a paired t test. Please note that this test was applied in an exploratory context and not for formal hypothesis testing since no previous data existed on which a power calculation could have been based. The statistical significance of potential sex differences in nocturnal changes, or in cartilage deformation after exercise was assessed using a Mann-Whitney $\mathrm{U}$ test (patella only).

\section{Results}

In all cartilage plates, a significant increase in cartilage thickness between evening (preexercise) and morning (preexercise) was observed. This amounted to $+2.4 \%$ in the patella, $+8.4 \%$ in the medial tibia and $+6.2 \%$ in the lateral tibia (table 1; fig. 1-3).

In the patella, the difference between evening postexercise and morning preexercise measurement was $+8.3 \pm$

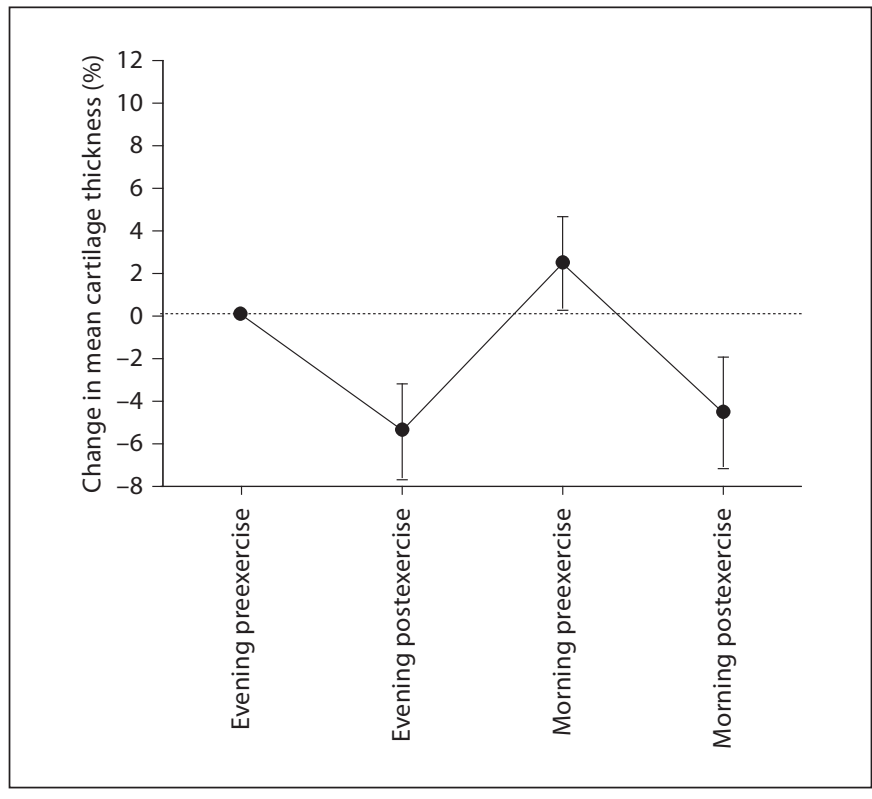

Fig. 1. Patella: changes ( $\%$ and standard deviation) in mean cartilage thickness after exercise (30 deep knee bends) in the morning and evening, and nocturnal changes in cartilage thickness.

$3.5 \%(\mathrm{p}<0.001)$, and there was a significant decrease in cartilage thickness postexercise, both in the morning and in the evening (table 1; fig. 1). The patellar deformation in the morning $(-6.8 \%)$ tended to be greater $(+28 \%)$ than that in the evening $(-5.4 \%)$, but the difference did not reach statistical significance $(\mathrm{p}=0.15)$.

In the medial tibia, the difference between evening postexercise and morning preexercise measurement was $+12.0 \pm 2.3 \%(\mathrm{p}<0.001)$. Again, a significant decrease in cartilage thickness was observed after static exercise in the morning and evening. As for the patella, deformation in the morning tended to be greater than that in the evening (+44\%; table 1; fig. 2), but the difference did not reach statistical significance $(\mathrm{p}=0.37)$.

In the lateral tibia, the difference between evening postexercise and morning preexercise measurement was $+10.3 \pm 3.9 \%(\mathrm{p}<0.001)$. A significant decrease in cartilage thickness was observed after exercise in the morning and evening. Again deformation in the morning tended to be greater than that in the evening ( $+38 \%$; table 1 ; fig. 3$)$ but the difference did not attain statistical significance $(\mathrm{p}=0.30)$.

When stratifying the results between both sexes, the increase in patellar cartilage thickness between evening (preexercise) and morning (preexercise) was similar in men $(+2.6 \pm 2.2 \%)$ and women $(+2.1 \pm 2.3 \%)$, with no 


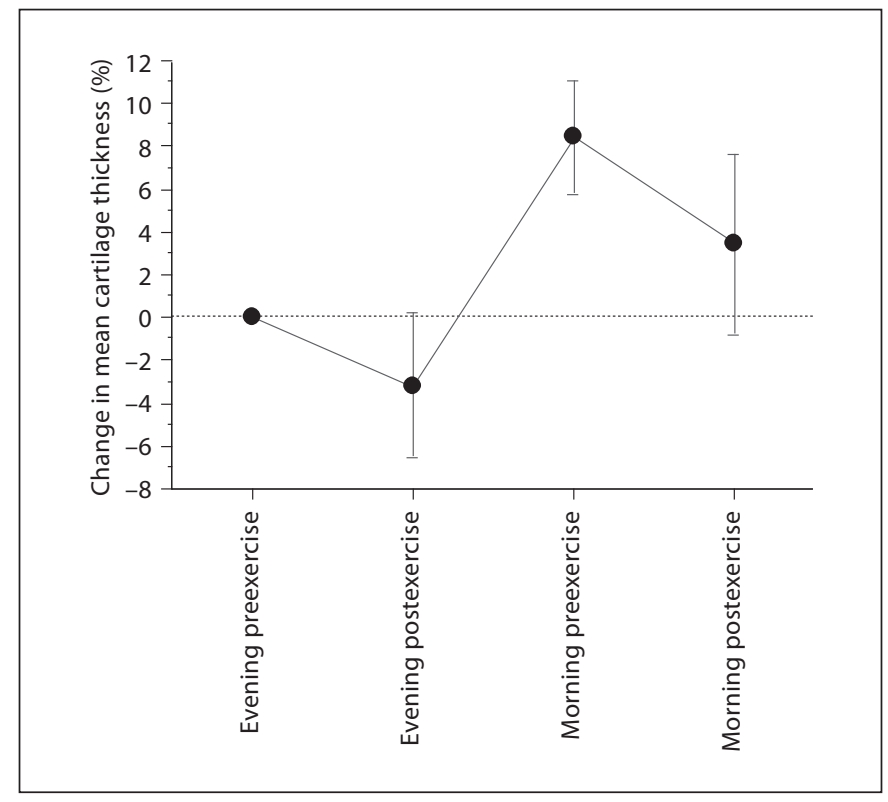

Fig. 2. Medial tibia: changes (\% and standard deviation) in mean cartilage thickness after exercise $(150 \%$ body weight, stance on one leg for $120 \mathrm{~s}$ ) in the morning and evening, and nocturnal changes in cartilage thickness.

significant difference in the increase between sexes $(\mathrm{p}=$ 0.56). Similarly, no relevant sex differences were identified for morning and evening postexercise deformation values (data not shown).

\section{Discussion}

This is the first study to explore nocturnal changes in (knee) cartilage thickness in vivo. As previous studies have shown that mechanical stimuli can enhance cell metabolism [Sah et al., 1989; Urban, 1994; Kim et al., 1995; Wong et al., 1997; Quinn et al., 1998; Buschmann et al., 1999; Ofek et al., 2010], the current findings may provide some guidance on regimens of mechanically stimulating cells in tissue culture, preparing them for the physiological in vivo demands in the target tissue to where they are transplanted. Although one must be careful in extrapolating these results from the healthy knees of young volunteers to osteoarthritic knees of elderly participants of clinical trials in a reciprocal manner (i.e. changes from morning to evening), the data may also provide clues as to whether baseline and follow-up measurements need to be matched for 'daytime' in clinical studies exploring cartilage loss (i.e. in osteoarthritis) over time [Eckstein et al., 2006a, 2009].

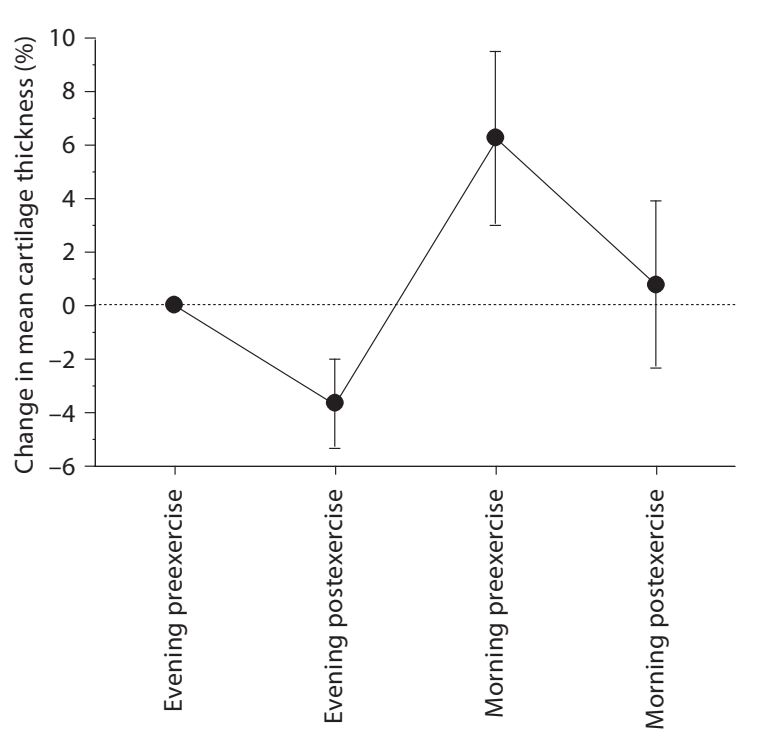

Fig. 3. Lateral tibia: changes (\% and standard deviation) in mean cartilage thickness after exercise (150\% body weight, stance on one leg for $120 \mathrm{~s}$ ) in the morning and evening, and nocturnal changes in cartilage thickness.

Limitations of the study include the small number of participants, particularly of the tibial cartilage deformation tests. Nocturnal changes in cartilage thickness (and changes after loading in the morning and evening), however, were statistically significant in all cartilage plates (patella, medial tibia and lateral tibia), showing that the study was sufficiently powered to address these. Although we could not statistically confirm with $95 \%$ confidence that morning postexercise deformation was greater than evening postexercise, the observations point in this direction and are physiologically plausible (see below). However, the preliminary observations from this exploratory study should be confirmed in larger cohorts. Further, they should be extended to participants with different degrees of knee osteoarthritis in order to explore to what extent the observations are clinically relevant.

A strength of this study is that postexercise acquisitions were not compared to a single preexercise acquisition, but to the mean of two preexercise acquisitions. Preexercise test-retest precision was evaluated for independent (nonpaired) readings and this compared favorably with the literature [Eckstein et al., 2006a]. This helped to reduce precision errors for the preexercise measurements. Therefore, the deformation measures in this study should be more robust than those based on single preexercise 
acquisitions [Eckstein et al., 2005]. The magnitude of patellar cartilage deformation observed in this study was similar to that seen in previous investigations using similar technology [Eckstein et al., 1998, 1999, 2000; Hudelmaier et al., 2001; Eckstein et al. 2005]. The tibial cartilage deformation observed after loading with $150 \%$ body weight was greater than that seen with MRI during static loading of one limb with $50 \%$ body weight, using an MRcompatible loading device [Cotofana et al., 2011]. However, it was similar to that seen after high-impact loading, and somewhat greater (and more uniform) than that previously reported after a static loading exercise with similar loading magnitudes to here [Eckstein et al., 2005]. This may be due to: (1) the tibial cartilage being measured after a combined exercise of deep knee bends and static loading; (2) a different setup of the static loading experiment incorporating the standing position which requires more balance and muscular cocontraction, and/or (3) smaller precision errors in the current study, due to the averaging of two preexercise acquisitions.

A previous study found no diurnal variation in the overall volume or thickness of femoral cartilage, but identified a thickness decrease in specific locations [Waterton et al., 2000]. Because the femur has relatively large surfaces that are not in contact with the tibia (or patella) during standing, the current study was confined to the tibial cartilage plates, of which relatively larger areas are mechanically loaded during knee bends or static loading. Our current findings extend previous observations [Waterton et al., 2000] in showing that knee cartilages undergo thickening overnight. Knee cartilage thickness in our study was compared after a normal day and directly after unloading overnight, whereas the above authors studied knees in the morning after the participants had walked from home to the MRI unit. The results of our study agree with those on intervertebral disc fibrocartilage that have shown an increase in height and volume of approximately $11 \%$ overnight during rest [Paajanen et al., 1994; Roberts et al., 1998; Malko et al., 2002; Hutton et al., 2003].

As cartilage contains $70-90 \%$ fluid, the thickening overnight is explained by fluid flow from the joint (or subchondral bone) to the interstitial matrix, whereas fluid is expelled from the matrix during loading [Mow et al., 1993; Ateshian et al., 1994; Eckstein et al., 1999; Herberhold et al., 1999]. Previous MRI-based studies were able to relate changes in hydration (and other compositional change) to cartilage deformation [Lusse et al., 2000; Mosher et al., 2005; Souza et al., 2010]. With more fluid being present in the matrix after periods of physical rest, there is also potential for a greater magnitude of exerciserelated deformation than in situations where the cartilage has just been loaded. Although patellar cartilage was between 2 and 3\% thicker in the morning than in the evening, previous studies have shown that simply walking leads to about $3 \%$ of patellar cartilage deformation [Eckstein et al., 2005]. Therefore, one may assume that it does not make a relevant difference whether patellar cartilage is imaged in the morning or evening in a longitudinal study, as long as the participants are not imaged after longer periods of unloading or severe exercise. Tibial cartilage, however, showed greater thickening (fluid influx) overnight and smaller deformation than the patella after loading (i.e. exercise). Therefore, the time of day of acquisition may play a stronger role in the assessment of femorotibial than patellar cartilage thickness in longitudinal studies [Eckstein et al., 2008]. This should be assessed by further experimental studies imaging femorotibial cartilage in the morning (after normal activity) and then again in the evening after a normal day.

In conclusion, this study shows that knee cartilage (thickness) recovers overnight by approximately $2-8 \%$, independent of sex. Given the lack of 'predeformation' and potentially greater hydration after nocturnal periods of unloading, morning postexercise deformation of the cartilage may have a greater magnitude than evening postexercise deformation.

\section{Acknowledgment}

This study was supported by funding from the German Research Foundation (DFG).
References

Nocturnal Cartilage Changes
Ateshian, G.A., W.M. Lai, W.B. Zhu, V.C. Mow (1994) An asymptotic solution for the contact of two biphasic cartilage layers. J Biomech 27: 1347-1360.

Buschmann, M.D., Y.J. Kim, M. Wong, E. Frank, E.B. Hunziker, A.J. Grodzinsky (1999) Stimulation of aggrecan synthesis in cartilage explants by cyclic loading is localized to re- gions of high interstitial fluid flow. Arch Biochem Biophys 366: 1-7.

Cotofana, S., F. Eckstein, W. Wirth, R.B. Souza, X. Li, B. Wyman, M.P. Hellio-Le Graverand, T. Link, S. Majumdar (2011) In vivo measures of cartilage deformation: patterns in healthy and osteoarthritic female knees using 3T MR imaging. Eur Radiol 21: 1127-1135. 
Eckstein, F., R.J. Buck, D. Burstein, H.C. Charles, J. Crim, M. Hudelmaier, D.J. Hunter, G. Hutchins, C. Jackson, V.B. Kraus, N.E. Lane, T.M. Link, L.S. Majumdar, S. Mazzuca, P.V. Prasad, T.J. Schnitzer, M.S. Taljanovic, A. Vaz, B. Wyman, M.P. Le Graverand (2008) Precision of 3.0 Tesla quantitative magnetic resonance imaging of cartilage morphology in a multicentre clinical trial. Ann Rheum Dis 67: 1683-1688.

Eckstein, F., D. Burstein, T.M. Link (2006a) Quantitative MRI of cartilage and bone: degenerative changes in osteoarthritis. NMR Biomed 19: 822-854.

Eckstein, F., A. Guermazi, F.W. Roemer (2009) Quantitative MR imaging of cartilage and trabecular bone in osteoarthritis. Radiol Clin North Am 47: 655-673.

Eckstein, F., M. Hudelmaier, R. Putz (2006b) The effects of exercise on human articular cartilage. J Anat 208: 491-512.

Eckstein, F., B. Lemberger, C. Gratzke, M. Hudelmaier, C. Glaser, K.H. Englmeier, M. Reiser (2005) In vivo cartilage deformation after different types of activity and its dependence on physical training status. Ann Rheum Dis 64: 291-295.

Eckstein, F., B. Lemberger, T. Stammberger, K.H. Englmeier, M. Reiser (2000) Patellar cartilage deformation in vivo after static versus dynamic loading. J Biomech 33: 819-825.

Eckstein, F., M. Reiser, K.H. Englmeier, R. Putz (2001) In vivo morphometry and functional analysis of human articular cartilage with quantitative magnetic resonance imaging from image to data, from data to theory. Anat Embryol (Berl) 203: 147-173.

Eckstein, F., M. Tieschky, S. Faber, K.H. Englmeier, M. Reiser (1999) Functional analysis of articular cartilage deformation, recovery, and fluid flow following dynamic exercise in vivo. Anat Embryol (Berl) 200: 419-424.

Eckstein, F., M. Tieschky, S.C. Faber, M. Haubner, H. Kolem, K.H. Englmeier, M. Reiser (1998) Effect of physical exercise on cartilage volume and thickness in vivo: MR imaging study. Radiology 207: 243-248.

Glüer, C.C., G. Blake, Y. Lu, B.A. Blunt, M. Jergas, H.K. Genant (1995) Accurate assessment of precision errors: how to measure the reproducibility of bone densitometry techniques. Osteoporos Int 5: 262-270.
Graichen, H., R. Eisenhart-Rothe, T. Vogl, K.H. Englmeier, F. Eckstein (2004) Quantitative assessment of cartilage status in osteoarthritis by quantitative magnetic resonance imaging: technical validation for use in analysis of cartilage volume and further morphologic parameters. Arthritis Rheum 50: 811-816.

Herberhold, C., S. Faber, T. Stammberger, M. Steinlechner, R. Putz, K.H. Englmeier, M. Reiser, F. Eckstein (1999) In situ measurement of articular cartilage deformation in intact femoropatellar joints under static loading. J Biomech 32: 1287-1295.

Hudelmaier, M., C. Glaser, J. Hohe, K.H. Englmeier, M. Reiser, R. Putz, F. Eckstein (2001) Age-related changes in the morphology and deformational behavior of knee joint cartilage. Arthritis Rheum 44: 2556-2561.

Hutton, W.C., J.A. Malko, W.A. Fajman (2003) Lumbar disc volume measured by MRI: effects of bed rest, horizontal exercise, and vertical loading. Aviat Space Environ Med 74: 73-78.

Kim, Y.J., L.J. Bonassar, A.J. Grodzinsky (1995) The role of cartilage streaming potential, fluid flow and pressure in the stimulation of chondrocyte biosynthesis during dynamic compression. J Biomech 28: 1055-1066.

Lusse, S., H. Claassen, T. Gehrke, J. Hassenpflug, M. Schunke, M. Heller, C.C. Gluer (2000) Evaluation of water content by spatially resolved transverse relaxation times of human articular cartilage. Magn Reson Imaging 18: 423-430.

Malko, J.A., W.C. Hutton, W.A. Fajman (2002) An in vivo MRI study of the changes in volume (and fluid content) of the lumbar intervertebral disc after overnight bed rest and during an 8-hour walking protocol. J Spinal Disord Tech 15: 157-163.

Mosher, T.J., H.E. Smith, C. Collins, Y. Liu, J. Hancy, B.J. Dardzinski, M.B. Smith (2005) Change in knee cartilage T2 at MR imaging after running: a feasibility study. Radiology 234: 245-249.

Mow, V.C., G.A. Ateshian, R.L. Spilker (1993) Biomechanics of diarthrodial joints: a review of twenty years of progress. J Biomech Eng 115: 460-467.

Ofek, G., E.P. Dowling, R.M. Raphael, J.P. McGarry, K.A. Athanasiou (2010) Biomechanics of single chondrocytes under direct shear. Biomech Model Mechanobiol 9: 153-162.
Paajanen, H., I. Lehto, A. Alanen, M. Erkintalo, M. Komu (1994) Diurnal fluid changes of lumbar discs measured indirectly by magnetic resonance imaging. J Orthop Res 12: 509-514.

Quinn, T.M., A.J. Grodzinsky, M.D. Buschmann, Y.J. Kim, E.B. Hunziker (1998) Mechanical compression alters proteoglycan deposition and matrix deformation around individual cells in cartilage explants. J Cell Sci 111: $573-$ 583.

Roberts, N., D. Hogg, G.H. Whitehouse, P. Dangerfield (1998) Quantitative analysis of diurnal variation in volume and water content of lumbar intervertebral discs. Clin Anat 11: $1-8$

Sah, R.L., Y.J. Kim, J.Y. Doong, A.J. Grodzinsky, A.H. Plaas, J.D. Sandy (1989) Biosynthetic response of cartilage explants to dynamic compression. J Orthop Res 7: 619-636.

Souza, R.B., C. Stehling, B.T. Wyman, M.P. Hellio Le Graverand, X. Li, T.M. Link, S. Majumdar (2010) The effects of acute loading on T1rho and T2 relaxation times of tibiofemoral articular cartilage. Osteoarthritis Cartilage 18: 1557-1563.

Stammberger, T., F. Eckstein, K.H. Englmeier, M. Reiser (1999a) Determination of 3D cartilage thickness data from MR imaging: computational method and reproducibility in the living. Magn Reson Med 41: 529-536.

Stammberger, T., F. Eckstein, M. Michaelis, K.H. Englmeier, M. Reiser (1999b) Interobserver reproducibility of quantitative cartilage measurements: comparison of B-spline snakes and manual segmentation. Magn Reson Imaging 17: 1033-1042.

Urban, J.P. (1994) The chondrocyte: a cell under pressure. Br J Rheumatol 33: 901-908.

Waterton, J.C., S. Solloway, J.E. Foster, M.C. Keen, S. Gandy, B.J. Middleton, R.A. Maciewicz, I. Watt, P.A. Dieppe, C.J. Taylor (2000) Diurnal variation in the femoral articular cartilage of the knee in young adult humans. Magn Reson Med 43: 126-132.

Wong, M., P. Wuethrich, M.D. Buschmann, P. Eggli, E. Hunziker (1997) Chondrocyte biosynthesis correlates with local tissue strain in statically compressed adult articular cartilage. J Orthop Res 15: 189-196. 